\title{
Peripheral blood miRNAs as a biomarker for chronic cardiovascular diseases
}

SUBJECT AREAS:

DIAGNOSTIC MARKERS

MIRNAS

ATHEROSCLEROSIS

Received

2 December 2013

Accepted

22 April 2014

Published

22 May 2014

Correspondence and requests for materials should be addressed to

Y.J. (jiangyongpost@

sina.com) or J.-H.C.

(leis428@yahoo.com)

\author{
Yong Jiang ', Hui-yan Wang ', Yan Li', Su-hong Guo', Lei Zhang' \& Jian-hui Cai ${ }^{2}$
}

'Laboratory Medical College, Jilin Medical College, Jilin, China, ${ }^{2}$ Department of surgery, Jilin Medical College, Jilin, China.
Vascular diseases are the most prevalent diseases worldwide. This study intended to analyze peripheral blood miRNA levels and their correlation with NT-pro-BNP and cTN-I in patients with atherosclerosis or pre-atherosclerotic conditions to build a dynamic correlation between vascular diseases and their biomarkers. Serum NT-pro-BNP and cTN-I levels were measured by their respective ELISA kits. The miRNA levels were assayed by quantitative PCR. Unique miRNA signatures were identified for both atherosclerosis and pre-atherosclerosis. The levels of miR-92a, 126, 130a, 222, and 370 levels were decreased in the peripheral blood of pre-atherosclerotic subjects. In atherosclerosis, miR-21, 122, 130a, and 211 were significantly increased whereas miR-92a, 126, and 222 were markedly decreased. Serum levels of NT-pro-BNP and cTN-I correlated with each other and increased with the progression of atherosclerosis. Moreover, the levels of cTN-I and NT-pro-BNP were positively correlated with miR-21 and negatively correlated with miR-126. Integrating specific pattern of miRNA levels with NT-pro-BNP and/or cardiac troponin may improve the diagnosis of cardiovascular diseases.

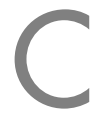
ardio-cerebrovascular disease (CVD) is the number one cause of mortality and morbidity worldwide $^{1}$. Obesity, diabetes, hyperlipidemia, and hypertension increasingly contribute to CVD mortality as the prevalence of those disorders increase over time ${ }^{2}$. Atherosclerosis is the most prominent indication among all CVDs, which is a chronic condition caused by lipid-induced inflammation of the vessel wall resulting from a complex interplay among endothelial cells, smooth muscle cells, macrophages, and leukocytes ${ }^{3}$. Atherosclerosis can remain asymptomatic for decades ${ }^{4}$ during the accumulation of fatty materials such as cholesterol and triglyceride on an artery wall until lumen stenosis is so severe that it restricts blood supply downstream to a level resulting in ischemia. The advancement of atherosclerosis are chronic, slowly progressive and cumulative. Diabetes, hyperlipidemia, and hypertension are the major risk factors promoting atherosclerosis ${ }^{4}$.

B-type natriuretic peptide (NT-pro-BNP) is a useful biomarker of acute heart failure (HF) in emergency settings ${ }^{5}$. The serum NT-pro-BNP level is correlated with the stage of $\mathrm{HF}^{5,6}$ and is associated with age, race ${ }^{7}$, and body mass index ${ }^{8}$. Moreover, it has been shown that serum NT-pro-BNP level is also increased in chronic renal dysfunction patients ${ }^{9,10}$.

Cardiac troponin I (cTN-I) is a prognostic indicator for acute myocardial infarction (AMI $)^{11,12}$. It has been shown that serum cTN-I level is elevated in patients with chronic pulmonary hypertension ${ }^{13}$, rhabdomyolysis ${ }^{14}$, and diabetic ketoacidosis ${ }^{15}$, indicating that the changes of NT-pro-BNP and cTN-I levels occurred before acute cardiovascular events.

MicroRNAs (miRNAs) belong to a class of small non-coding RNA molecules. MiRNAs bind to the $3^{\prime}$ untranslated regions of messenger RNAs to block translation and/or to promote mRNA degradation ${ }^{16}$. MiRNA has been shown to regulate physiological and pathophysiological process including cardiac regeneration $^{17}$ and atherosclerosis ${ }^{18}$. Circulating miRNAs have emerged as biomarkers for different diseases ${ }^{19-22}$. As most studies focused on the association between changes of miRNAs with a clearly defined stage of a disease, we intended to investigate both physiological and miRNA changes of patients with atherosclerosis or with conditions leading to atherosclerosis.

\section{Results}

Changes of NT-pro-BNP and cTN-I in patients with pre-atherosclerotic conditions or atherosclerosis. NT-pro-BNP and cTN-I serum concentration increased in atherosclerotic (Athero) patients and preatherosclerotic (pre-Athero) patients (patients with hyperlipidemia, hypertension, and or/diabetes). The average serum NT-pro-BNP level was increased to $42.77 \mathrm{pg} / \mathrm{ml}$ and $49.17 \mathrm{pg} / \mathrm{ml}$ in pre-atherosclerotic and atherosclerotic patients respectively from $30.15 \mathrm{pg} / \mathrm{ml}$ in normal controls $(\mathrm{p}<0.05$ Athero vs control and 
pre-Athero vs control; $\mathrm{p}<0.05$ Athero vs pre-Athero) (Fig. 1A). The serum cTN-I concentration was increased from $10.24 \mathrm{pg} / \mathrm{ml}$ in controls to $12.02 \mathrm{pg} / \mathrm{ml}$ in pre-atherosclerotic patients and $13.14 \mathrm{pg} / \mathrm{ml}$ in athersclerotic patients $(\mathrm{p}<0.05$ vs control and $\mathrm{p}$ $<0.05$ vs pre-athero) (Fig. 1B).

The serum levels of NT-pro-BNP and cTN-I were highly correlated with each other in the subjects overall $\left(\mathrm{R}^{2}=0.8229\right.$; Fig. $\left.2 \mathrm{~A}\right)$. When stratified by healthy controls, pre-atherosclerotic, and atherosclerotic groups, the highest correlation between NT-pro-BNP and cTN-I was seen in healthy individuals $\left(\mathrm{R}^{2}=0.9593\right.$, Fig. $\left.2 \mathrm{~B}\right)$, and became weaker in pre-atherosclerotic $\left(\mathrm{R}^{2}=0.7046\right.$, Fig. $\left.2 \mathrm{C}\right)$ and atherosclerotic $\left(\mathrm{R}^{2}=0.716\right.$, Fig. $\left.2 \mathrm{D}\right)$ patients.

Signatures of miRNA expression in pre-atherosclerotic and atherosclerotic patients. The profiles of a selected group of miRNAs in peripheral blood were significantly different in patient group with atherosclerosis or without atherosclerosis but having hypertension, diabetes, and/or hyperlipidemia from that of healthy individuals (Fig. 3). Specifically, miR-92a, miR-126, miR-130a, miR-222, and miR-370 were markedly reduced in pre-atherosclerotic patients, with miR-126 and miR-130a reduced more than 50\% (Fig. 3A). However, The levels of miR-21, miR-122, miR-130a, and miR-211 in peripheral blood significantly increased whereas those of miR-92a, miR-126, and miR-222 markedly decreased in atherosclerotic patients (Fig 3B).

The peripheral blood levels of miR-21 and miR-122 in patients with hyperlipidemia, diabetes, and/or hypertension were comparable to that of the control group but significantly increased with atherosclerosis (Fig. 4) though miR-21 showed a trend of increasing with the emergency of atherosclerosis. On the other hand, the level of miR-130a was significantly increased in the atherosclerosis group whereas it was deeply depressed in pre-atherosclerotic patients ( $p<0.01$ Athero vs control, pre-Athero vs control, and Athero vs pre-Athero) (Fig. 4).

Levels of miR-21 and miR-126 are well correlated with NT-proBNP and cTN-I levels. The correlation between the expression of individual miRNA and NT-pro-BNP or cTN-I level was analyzed. A negative correlation was identified between miR-126 with cTN-I ( $\mathrm{R}^{2}$ $=0.6758$; Fig. 5A) and NT-pro-BNP $\left(\mathrm{R}^{2}=0.8317\right.$; Fig. $\left.5 \mathrm{~B}\right)$ levels whereas a positive correlation between miR-21 with cTN-I level $\left(\mathrm{R}^{2}\right.$ $=0.7066$; Fig. 5C) and NT-pro-BNP level $\left(\mathrm{R}^{2}=8601\right.$; Fig. 5D) among atherosclerotic patients (Fig. 5B and 5D) was observed.

\section{Discussion}

A miRNA expression signature emerged from patients both with atherosclerosis or without atherosclerosis but diagnosed with one or more disorders of hyperlipidemia, diabetes mellitus, and hypertension. The levels of NT-pro-BNP and TN, considered markers for acute coronary artery diseases and heart failure, were increased in those patients.

The dysregulation of miRNA has been widely reported in different cardiac and vascular diseases ${ }^{18,21-23}$. The peripheral blood levels of miR-92a, miR-126, and miR-222 in this study were markedly decreased in both atherosclerotic and pre-atherosclerotic patients compared to healthy controls though the decease of miR-92a and miR-222 in pre-atherosclerotic patients was not as significant as that in atherosclerotic patients. Endothelial miR-126 inhibited VCAM-1 protein levels during inflammation ${ }^{24}$ to protect against the onset of atherosclerosis. MicroRNA-92a regulated Krüppel-Like factors 4 and 2 in arterial endothelium and contributed to regional atherosusceptibility and protection in vivo ${ }^{25}$ and its circulating level was found significantly lower in patients with coronary artery disease ${ }^{26}$. The opposite effects of miR-221/222 on the proliferation, migration, and apoptosis of endothelial cells and vascular smooth muscle cells allowed them to promote neointimal formation while inhibit reendothelialization after vascular injury ${ }^{27}$. The levels of miR-221 and miR-222 were decreased whereas levels of miR-21, miR-130a, and some other miRNAs in patients with peripheral arterial disease $\mathrm{e}^{28}$. Interestingly, plasma level of liver specific miR-122 was increased significantly after cardiogenic shock ${ }^{29}$.

There have not been many efforts to establish the association between specific miRNA and NT-pro-BNP or troponin to develop a multi-factor diagnostic panel for various vascular diseases. In the present study, a stronger association was found between NT-proBNP with miR-126 and with miR-21 in atherosclerotic patients while cTN-I showed good association with those two microRNAs as well. However, due to the limited number of patients with hypertension, diabetes, and/or hyperlipidemia, it was impossible to further stratify the data from the pre-atherosclerotic group. A correlation between cTN-I and peripheral blood miR-133a, miR-208a, and especially miR-133b was reported during early myocardial injury and recovery in patients after heart transplantation ${ }^{30}$, indicating that circulating miRNA and cTN-I levels could serve as an indicator of cardiac injury. The levels of cTN-T, miR-499, along with other risk factors such as hypercholesterolemia, diabetes, and smoking were able to predict the mortality of patients with acute myocardial infarction ${ }^{31}$. The incorporation of microRNA levels has been shown to improve the diagnostic power of NT-pro-BNP ${ }^{32}$. Those data demonstrated the possibility to identify the correlations between specific miRNA signature with NT-pro-BNP and/or cardiac troponin and to improve the diagnostic power.

In conclusion, the peripheral miRNA signature was different in patients with atherosclerosis or with pre-atherosclerotic conditions. Plasma NT-pro-BNP and cTN-I levels showed a trend of increase
A

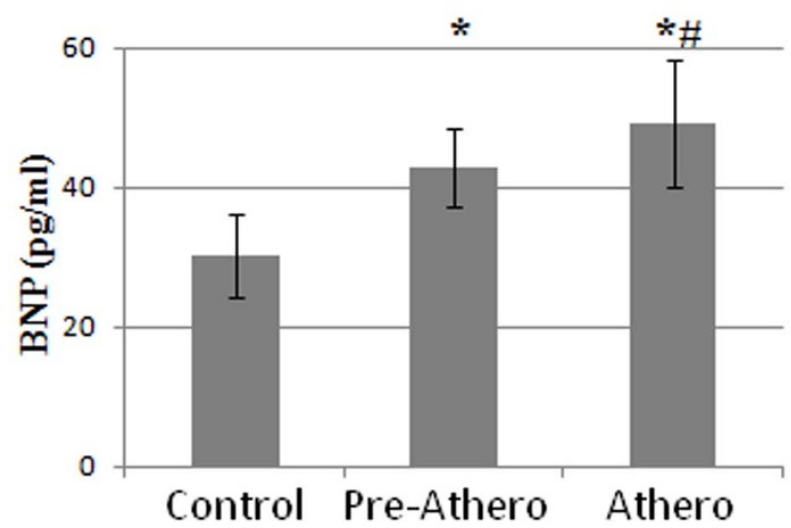

B

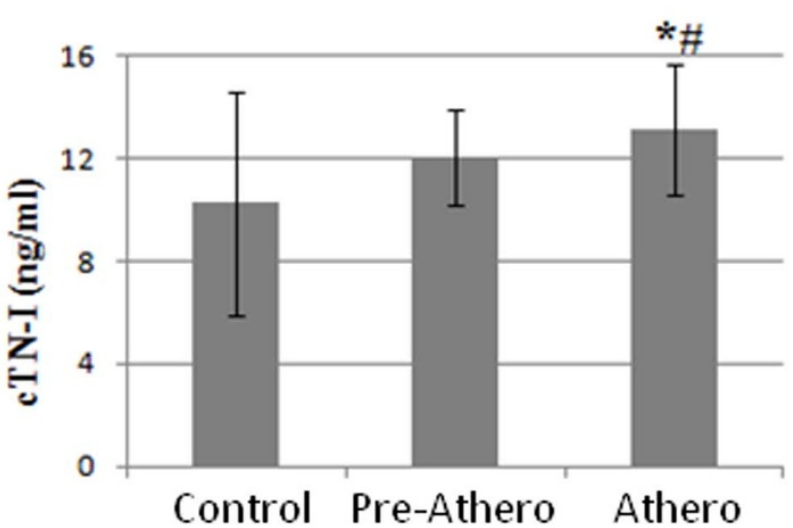

Figure $1 \mid$ The serum NT-pro-BNP and cTN-I levels were increased in both atherosclerotic and pre-atherosclerotic patients. Serum levels of NT-proBNP (A) and cTN-I (B) from cardiovascular disease patients and healthy controls were analyzed by ELISA kits. 
A

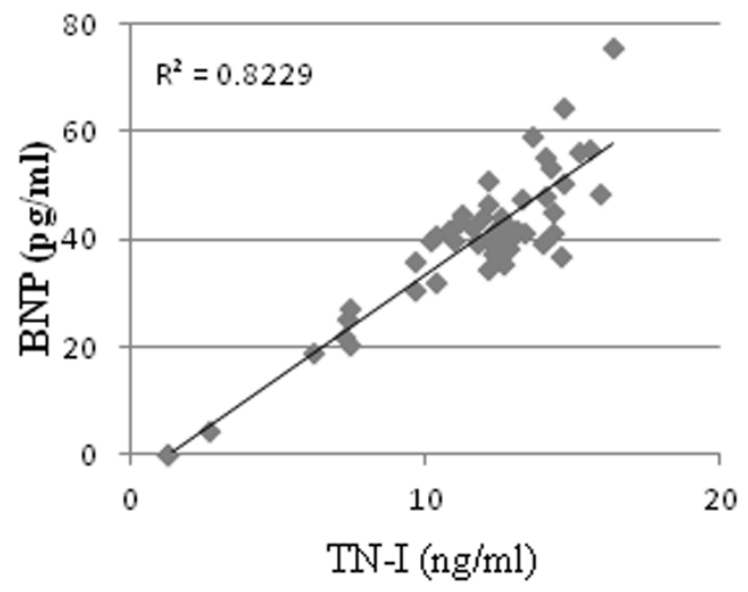

C

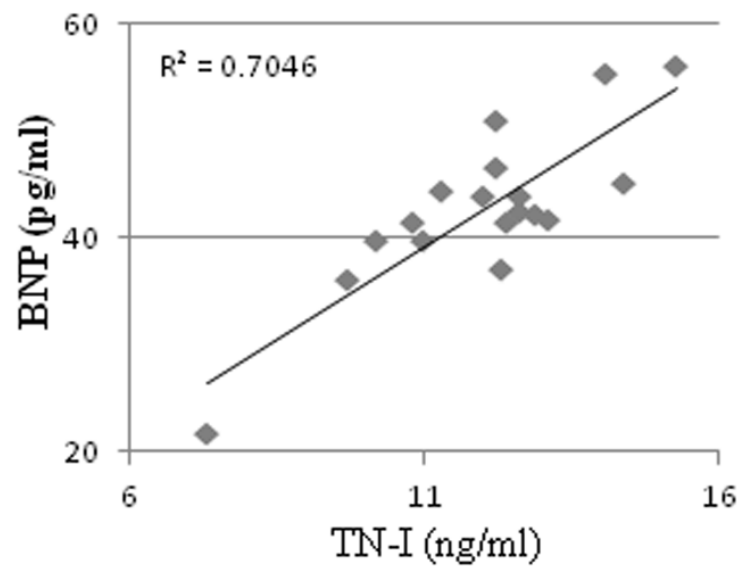

B

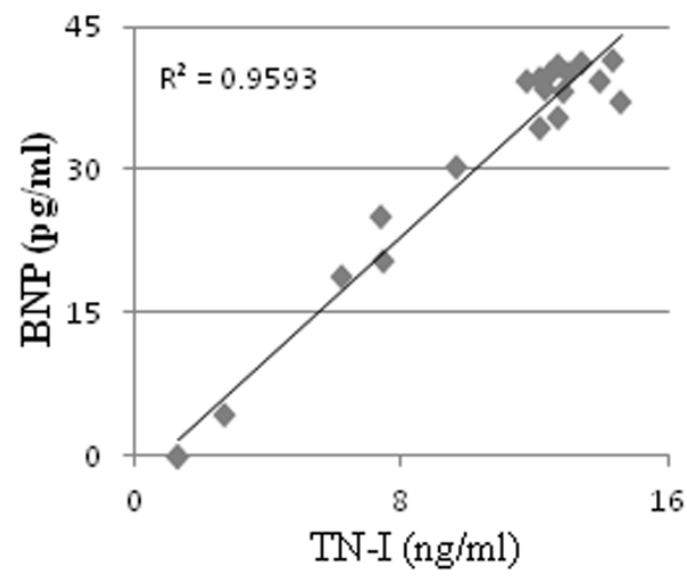

D

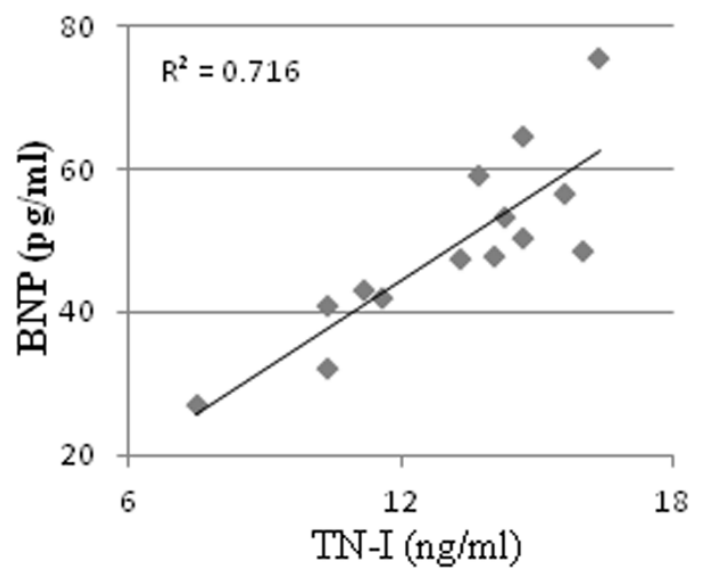

Figure 2 The serum NT-pro-BNP and cTN-I levels were positively correlated. Strong correlation between serum NT-pro-BNP and cTN-I levels was found in all study subjects (A), controls (B), pre-atherosclerotic (C), and atherosclerotic (D) groups.

with the progression of atherosclerosis and correlated with each other. Meanwhile, strong association of miR-21 and miR-126 with NTpro-BNP and cTN-I was identified in patients with atherosclerosis, indicating that integration of miRNA signature with other parameters like NT-pro-BNP and cTN could significantly improve the diagnostic power of vascular diseases.
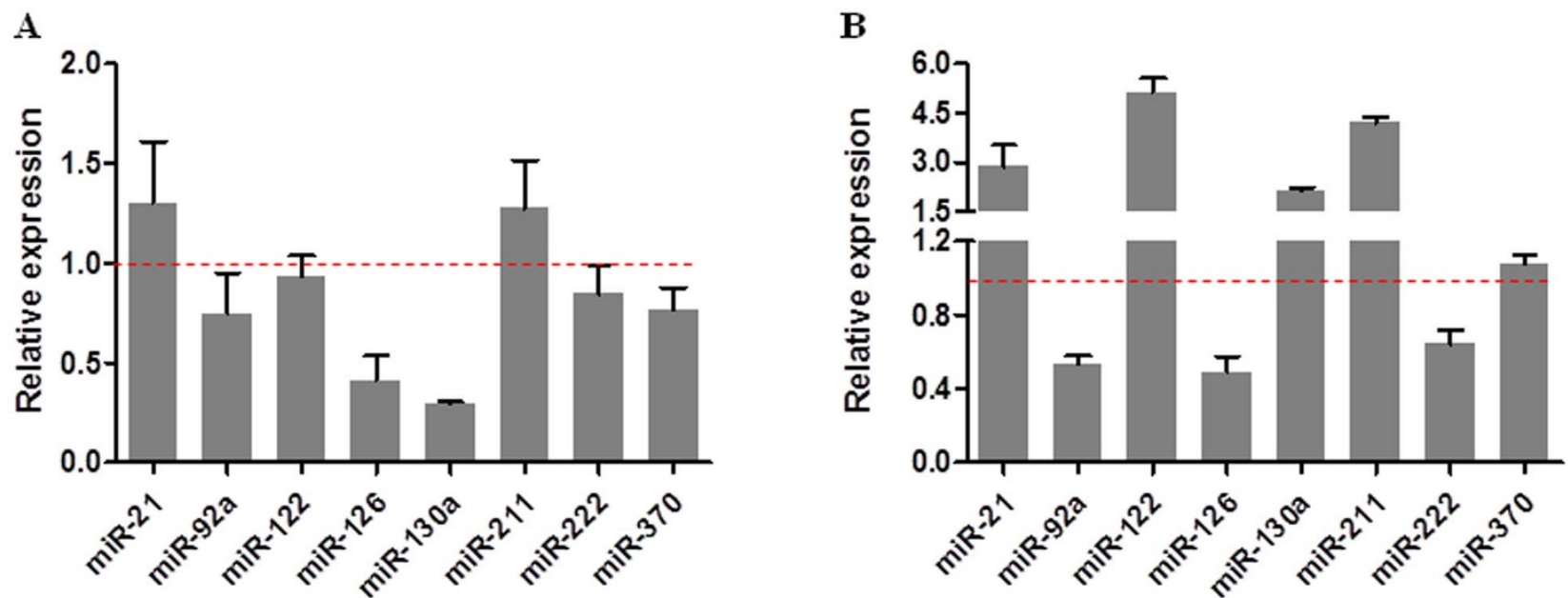

Figure 3 | Unique peripheral blood miRNA signatures distinguish pre-atherosclerosis and atherosclerosis. The miRNA level in peripheral blood was assayed by reverse transcription quantitative real-time PCR. The pattern of miRNA of pre-atherosclerotic (A) and atherosclerotic (B) patients were distinct. 


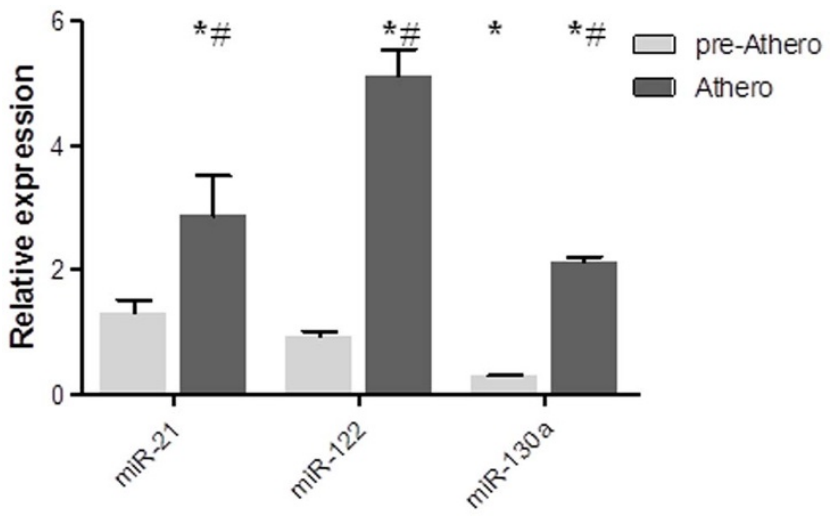

Figure $4 \mid$ The changes of miR-21, miR-122, and miR-130a associated with pre-atherosclerotic conditions and atherosclerosis. *: $\mathrm{p}<0.05$ vs control; \#: $\mathrm{p}<0.05$ vs pre-atherosclerosis.

\section{Methods}

Study Subjects. Subjects, 20 healthy control, 20 patients with atherosclerosis, and 20 patients with one or more indications of hypertension, hyperlipidemia, and diabetes mellitus but without atherosclerosis detected, were recruited from January 2013 through June 2013 at the affiliate hospital of Jilin Medical University. All procedures conformed with the Declaration of Helsinki and approved by the institutional review board of Jilin Medical University. A signed consent form was obtained from each participant. All hypertension, hyperlipidemia, and diabetes patients undergone coronary calcium scoring by CT, carotid intimal media thickness measurement by ultrasound, lipoprotein subclass analysis, and $\mathrm{HbAlc}$, hsC-reactive protein, and homocysteine measurements to detect the existence of atherosclerosis.

\begin{tabular}{|ll|}
\hline Table 1 | Nucleotide sequence & \\
miR-21 & TGCCGCCAACACCAGTCGAT \\
miR-92a & TGCCGCAGGTGGGATCGGT \\
miR-122 & TGCCGCAACGCCATTATCACA \\
miR-126 & TGCCGCTCGTACCGTGAGTAA \\
miR-130a & TGGCCAATGTTAAAAGGGCAT \\
miR-21 1 & TGCCGCGCAGGGACAGCAAA \\
miR-222 & TGCCGCAGCTACATCTGGCT \\
hiR-370 & TGCCGCGCCTGCTGGGGTG \\
& TGCTGGATGACACGCAAATTC \\
\hline
\end{tabular}

Blood Sample Processing. Fasting blood $(2 \mathrm{ml})$ was drawn from each participant into blood collection tube containing sodium heparin (Nihoo International, Wenzhou, China) and put on ice immediately and processed within $3 \mathrm{hr}$ after drawing. One milliliter of blood was transferred into a eppendorf tubes and centrifuged at $1000 \mathrm{rpm}$ for $5 \mathrm{~min}$. $200 \mu \mathrm{l}$ serum was set aside for NT-pro-BNP and cTN-I analyses. The total RNA was isolated from $1 \mathrm{ml}$ whole blood sample using Trizol reagent (TransGen, Nanjing, China) according to manufactures' protocols.

Serum NT-pro-BNP and cTN-I level measurement. Serum NT-pro-BNP and cTN-I levels were analyzed with ELISA kits from Yupingbio (Shanghai, China) according to kit protocols. Briefly, $50 \mu \mathrm{l}$ of standards and samples (1:4 diluted with sample diluents) was added into assay strips followed by mixing in $100 \mu \mathrm{l}$ of HRP-conjugated detecting antibody. The strips were sealed and incubated at $37^{\circ} \mathrm{C}$ for $60 \mathrm{~min}$, washed 5 times with $1 \mathrm{ml}$ per well of washing solution, patted dry, added $50 \mu \mathrm{l}$ each of substrates A and B followed by incubating at $37^{\circ} \mathrm{C}$ for $15 \mathrm{~min}$ and then stopped with $50 \mu \mathrm{l}$ of Stop Solution and read at $450 \mathrm{~nm}$ within $15 \mathrm{~min}$. Sample NT-pro-BNP and cTN-I concentrations were calculated against its own standard curve.

Real-time quantitative analysis of miRNA. The first strand cDNA of small RNAs was synthesized using a one step simultaneous polyadenylation and reverse

B

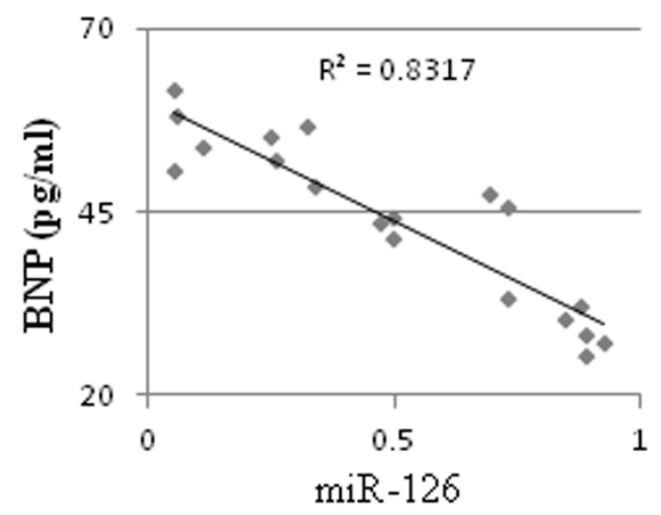

D

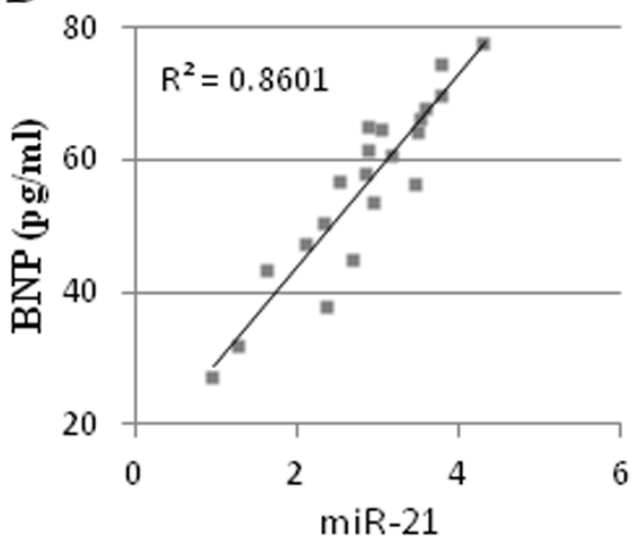

Figure $5 \mid$ The miR-21 and miR-126 levels were strongly associated with NT-pro-BNP and cTN-I in atherosclerosis. MiR-126 was negatively correlated with cTN-I (A) and NT-pro-BNP (B) levels while miR-21 was positively associated with cTN-I (C) and NT-pro-BNP (D) levels. 
transcription system from Chi Biotechnology (Jiangyin, China) according to manufacturer's instruction. A panel of 8 miRNAs (Table 1) linked to cardiovascular diseases were analyzed with MirCount ${ }^{\mathrm{TM}}$ system (Chi Biotechnology, Jiangyin, China) following supplier's protocol. The relative expression levels were calculated by $2^{-\Delta \Delta \mathrm{Ct}}$ method with U6 as internal control.

Statistical analyses. The correlation between different factors, linear regression, and co-efficiency were analyzed by Graphpad Prism 5. The difference between groups was analyzed by t-test with either Graphpad Prism 5 or Excel. It was considered statistically significant if $\mathrm{p}<0.05$.

1. Shah, A. S. et al. Global association of air pollution and heart failure: a systematic review and meta-analysis. Lancet 382, 1039-1048 (2013).

2. Go, A. S. et al. Executive summary: heart disease and stroke statistics--2013 update: a report from the American Heart Association. Circulation 127, 143-152 (2013).

3. Wei, Y., Nazari-Jahantigh, M., Neth, P., Weber, C. \& Schober, A. MicroRNA-126, -145, and -155: a therapeutic triad in atherosclerosis? Arterioscler Thromb Vasc Biol 33, 449-454 (2013).

4. Ross, R. The pathogenesis of atherosclerosis: a perspective for the 1990s. Nature 362, 801-809 (1993).

5. Maisel, A. S. et al. Rapid measurement of B-type natriuretic peptide in the emergency diagnosis of heart failure. N Engl J Med 347, 161-167 (2002).

6. Tsutamoto, T. et al. Attenuation of compensation of endogenous cardiac natriuretic peptide system in chronic heart failure: prognostic role of plasma brain natriuretic peptide concentration in patients with chronic symptomatic left ventricular dysfunction. Circulation. 96, 509-516 (1997).

7. Maisel, A. S. et al. Impact of age, race, and sex on the ability of B-type natriuretic peptide to aid in the emergency diagnosis of heart failure: results from the Breathing Not Properly (BNP) multinational study. Am Heart J 147, 1078-1084 (2004).

8. Daniels, L. B. et al. How obesity affects the cut-points for B-type natriuretic peptide in the diagnosis of acute heart failure. Results from the Breathing Not Properly Multinational Study. Am Heart J 151, 999-1005 (2006).

9. Vickery, S. et al. B-type natriuretic peptide (BNP) and amino-terminal pro-BNP in patients with CKD: relationship to renal function and left ventricular hypertrophy. Am J Kidney Dis 46, 610-620 (2005).

10. Jafri, L. et al. B-type natriuretic peptide versus amino terminal pro-B type natriuretic peptide: selecting the optimal heart failure marker in patients with impaired kidney function. BMC Nephrol 14, 117 (2013).

11. Tanasijevic, M. J., Cannon, C. P. \& Antman, E. M. The role of cardiac troponin-I (cTnI) in risk stratification of patients with unstable coronary artery disease. Clin Cardiol 22, 13-16 (1999).

12. Tsutamoto, T. et al. Prognostic role of highly sensitive cardiac troponin I in patients with systolic heart failure. Am Heart J 159, 63-67 (2010).

13. Vélez-Martínez, M. et al. Association of cardiac troponin I with disease severity and outcomes in patients with pulmonary hypertension. Am J Cardiol 111, 1812-1817 (2013)

14. Punukollu, G. et al. Elevated serum cardiac troponin I in rhabdomyolysis. Int Cardiol 96, 35-40 (2004).

15. Abdo, A. S. \& Geraci, S. A. Significance of elevated cardiac troponin I in patients with diabetic ketoacidosis. J Miss State Med Assoc 54, 127-130 (2013).

16. Carthew, R. W. \& Sontheimer, E. J. Origins and Mechanisms of miRNAs and siRNAs. Cell 136, 642-655 (2009).

17. Eulalio, A. et al. Functional screening identifies miRNAs inducing cardiac regeneration. Nature 492, 376-381 (2012).

18. Hergenreider, E. et al. Atheroprotective communication between endothelial cells and smooth muscle cells through miRNAs. Nat Cell Biol 14, 249-256 (2012)

19. Dorval, V., Nelson, P. T. \& Hébert, S. S. Circulating microRNAs in Alzheimer's disease: the search for novel biomarkers. Front Mol Neurosci 6, 24 (2013).

20. Sita-Lumsden, A., Dart, D. A., Waxman, J. \& Bevan, C. L. Circulating microRNAs as potential new biomarkers for prostate cancer. Br J Cancer 108, 1925-1930 (2013)
21. van Empel, V. P., De Windt, L. J. \& da Costa Martins, P. A. Circulating miRNAs: reflecting or affecting cardiovascular disease? Curr Hypertens Rep 14, 498-509 (2012).

22. Kuster, D. W. et al. MicroRNA transcriptome profiling in cardiac tissue of hypertrophic cardiomyopathy patients with MYBPC3 mutations. J Mol Cell Cardiol 65C, 59-66 (2013).

23. Karunakaran, D. \& Rayner, K. J. MicroRNAs in Cardiovascular Health: From Order to Disorder. Endocrinology 154, 4000-4009 (2013).

24. Asgeirsdóttir, S. A. et al. MicroRNA-126 contributes to renal microvascular heterogeneity of VCAM-1 protein expression in acute inflammation. Am J Physiol Renal Physiol 302, F1630-1639 (2012).

25. Fang, Y. \& Davies, P. F. Site-specific microRNA-92a regulation of Kruppel-like factors 4 and 2 in atherosusceptible endothelium. Arterioscler Thromb Vasc Biol 32, 979-987 (2012)

26. Fichtlscherer, S. et al. Circulating microRNAs in patients with coronary artery disease. Circ Res 107, 677-684 (2010).

27. Liu, X., Cheng, Y., Yang, J., Xu, L. \& Zhang, C. Cell-specific effects of miR-221/222 in vessels: molecular mechanism and therapeutic application. J Mol Cell Cardiol 52, 245-255 (2012).

28. $\mathrm{Li}, \mathrm{T}$. Identification of miR-130a, miR-27b and miR-210 as serum biomarkers for atherosclerosis obliterans. Clin Chim Acta 412, 66-70 (2011).

29. Andersson, P. et al. Plasma levels of liver-specific miR-122 is massively increased in a porcine cardiogenic shock model and attenuated by hypothermia. Shock 37, 234-238 (2012)

30. Wang, E. et al. Circulating miRNAs reflect early myocardial injury and recovery after heart transplantation. J Cardiothorac Surg 8, 165 (2013).

31. Goretti, E., Vausort, M., Wagner, D. R. \& Devaux, Y. Association between circulating microRNAs, cardiovascular risk factors and outcome in patients with acute myocardial infarction. Int J Cardiol 168, 4548-4550 (2013).

32. Ellis, K. L. et al. Circulating microRNAs as candidate markers to distinguish heart failure in breathless patients. Eur J Heart Fail 15, 1138-1147 (2013).

\section{Acknowledgments}

The authors would like to thank Hong-mei Cao, Chun-yan Wang, Hao Wang and Lei Li for technical help. We also thank Dr. Shi Lei (Puhe Biotechnologies) for critical reading. This work was supported by the National Natural Science Fundation of China (Grant No. 31100566), Chunmiao Cultivation Program for University Talents in Jilin Province (2013-352), Youth Research Fund Project of Science and Technology Department of Jilin Province (20140520006JH), the Training Program for Outstanding Young Talents in Jilin, Jilin Provincial Health Department Project (Grant No. 2011ZC029; 2011Z089).

\section{Author contributions}

Y.J. and J.H.C. conceived the study and wrote the manuscript; H.W., Y.L., S.G. and L.Z. generated data and reviewed the manuscript.

\section{Additional information}

Competing financial interests: The authors declare no competing financial interests.

How to cite this article: Jiang, Y. et al. Peripheral blood miRNAs as a biomarker for chronic cardiovascular diseases. Sci. Rep. 4, 5026; DOI:10.1038/srep05026 (2014).

This work is licensed under a Creative Commons Attribution-NonCommercialNoDerivs 4.0 International License. The images or other third party material in this article are included in the article's Creative Commons license, unless indicated otherwise in the credit line; if the material is not included under the Creative Commons license, users will need to obtain permission from the license holder in order to reproduce the material. To view a copy of this license, visit http:// creativecommons.org/licenses/by-nc-nd/4.0/ 
SUBJECT AREAS: DIAGNOSTIC MARKERS MIRNAS

ATHEROSCLEROSIS

\section{SCIENTIFIC REPORTS:}

$4: 5026$

DOI: $10.1038 /$ srep05026

(2014)

Published: 22 May 2014

Updated: 12 March 2015

Correspondence and requests for materials should be addressed to

Y.J. (jiangyongpost@ sina.com) or J.-H.C. (leis428@yahoo.com).

\section{CORRIGENDUM: Peripheral blood miRNAs as a biomarker for chronic cardiovascular diseases}

\author{
Yong Jiang', Hui-yan Wang', Yan Li', Su-hong Guo', Lei Zhang' \& Jian-hui Cai' \\ 'Laboratory Medical College, Jilin Medical College, Jilin, China, ${ }^{2}$ Department of surgery, Jilin Medical College, Jilin, China.
}

To better reflect the contributions to this study, the authors wish to make the following changes to the Article.

'Yong Jiang, Hui-yan Wang, Hong-mei Cao, Chun-yan Wang, Lei Zhang, Hao Wang, Lei Liu, Yan Li, \& Jian-hui Cai'

Now reads

'Yong Jiang, Hui-yan Wang, Yan Li, Su-hong Guo, Lei Zhang \& Jian-hui Cai'

\section{Acknowledgements}

'The authors would like to thank Dr. Shi Lei (Chi Biotechnology) for critical reading of the manuscript. This work was supported by grants from the Jilin Provincial Health Department Project (2011ZC029), National Natural Science Foundation of China $(31100566 ; 81273421 ; 81202953 ; 81100808)$, Scientific research of Jilin province college talent cultivation project of Chunmiao (2013-352), and Jilin City Science and Technology Project (201032244)'

Now reads

'The authors would like to thank Hong-mei Cao, Chun-yan Wang, Hao Wang and Lei Li for technical help. We also thank Dr. Shi Lei (Puhe Biotechnologies) for critical reading. This work was supported by the National Natural Science Fundation of China (Grant No. 31100566), Chunmiao Cultivation Program for University Talents in Jilin Province (2013-352), Youth Research Fund Project of Science and Technology Department of Jilin Province (20140520006JH), the Training Program for Outstanding Young Talents in Jilin, Jilin Provincial Health Department Project (Grant No. 2011ZC029; 2011Z089).'

\section{Author Contributions Statement}

'Y.J. and J.H.C. conceived the study and wrote the manuscript; H.Y.W., H.M.C., C.Y.W., L.Z., H.W., L.L. and Y.L. generated data and reviewed the manuscript.'

Now reads

'YJ and JHC conceived the study and wrote the manuscript; HW, YL, SG, and LZ generated data and reviewed the manuscript.'

These changes have been made in the PDF and HTML versions of the Article. 